\title{
A new method for the direct tracking of in vivo lignin nanocapsules in Eragrostis tef (Poaceae) tissues
}

\author{
Sara Falsini, ${ }^{1,2}$ Corrado Tani, ${ }^{1}$ Silvia Schiff, ${ }^{1}$ Cristina Gonnelli, ${ }^{1}$ Ilaria Clemente, ${ }^{2,3}$ Sandra Ristori, ${ }^{2}$ \\ Alessio Papini, ${ }^{1,4}$ \\ ${ }^{1}$ Department of Biology, University of Florence \\ ${ }^{2}$ Department of Chemistry "Ugo Schiff” \& CSGI, University of Florence, Sesto Fiorentino (FI) \\ ${ }^{3}$ Department of Biotechnology, Chemistry and Pharmacy, University of Siena \\ ${ }^{4}$ CSET, Centro Studi Erbario Tropicale, Florence, Italy
}

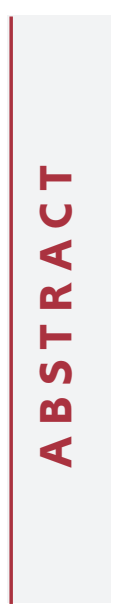

\begin{abstract}
Environmental concerns have driven scientists to research new eco-friendly approaches for the preparation of nanosystems. For this purpose, novel bio-polymers have been selected. Among these, one of the most promising is lignin, which is biodegradable and biocompatible. Additionally, lignin is one of the main by-products of the paper industry and can be re-used in nanosystems building. Lignin-based nanosystems could be used in agriculture, to improve the uptake of bioactive compounds, thus avoiding soil pollution. However, the mechanism of penetration in the plant and the route of transportation within the internal plant tissues are unknown and need to be clearly elucidated. Here we present a method of lignin nanocapsules staining and tracking by fluorochrome: Fluoral Yellow 088, which is a well-suited dye for the tracking of lipids and other oil phases. Two different applications were applied: in the first one fourteen-day plants were soaked with fluorescent nanocapsules (fNCs) pure solution and in the second one, Eragrostis tef plants were laid down on blotting paper and soaked with diluted fNCs solution. Wetting the roots of Teff plantlets with the pure fNCs solution resulted in the most efficient way of nanocapsule entrance. The dyeing of lignin nanocapsules allowed us to track them in Eragrostis tef plant tissues through microscopic observations. In particular, fNCs were proven to be able to permeate roots, reaching xylem vessels where, through water pressure, they reached the leaf.
\end{abstract}

Key words: Nanocapsule tracking; Fluoral Yellow 088; Eragrostis tef; nanocapsule histochemistry; nanotechnology; phytonanotechnology.

Correspondence: Alessio Papini, Department of Biology, University of Florence, Via Micheli 1-3, 50121 Florence, Italy. E-mail: alpapini@unifi.it

Contributions: All the authors made a substantive intellectual contribution. All the authors have read and approved the final version of the manuscript and agreed to be accountable for all aspects of the work. 


\section{Introduction}

Nanotechnology has the potential to be used in a wide range of applications, among which medicine, engineering and energy. The opportunity to modify the physicochemical properties of nanoassemblies (size, shape, surface structure, etc.) makes them suitable for such different applications. More recently, nanomaterials have found their use also in agriculture, where they have been designed for the delivery of active biomolecules such as pesticides, hormones and herbicides. ${ }^{1-4}$ However, the growing interest of nanotechnology applications in agriculture ${ }^{5,6}$ has opened a new conception that some authors called "Phytonanotechnology". 7 This new application is aiming at the development of nanotechnology at large-scale production, although the perspective to extend the use of nanovectors has risen many concerns in the scientific community about their eventual release in the environment. In fact, once applied in the agricultural field, nanosystems may pollute the soil and diffuse in neighboring areas, possibly penetrating the groundwater. Besides, the long-term fate of nanomaterials is difficult to forecast, mainly due to their size, which makes tracking of nanoparticles in the environment extremely difficult. ${ }^{8,9}$ Therefore, new sustainable approaches are needed to overcome the possible contamination of water and soil, especially if nanovectors are planned to be used at large scale. For this reason, industries are developing eco-friendly strategies to reduce the impact not only during nanomaterial production processes, ${ }^{10}$ but also in a perspective of their release in the environment. ${ }^{11,12}$ Moreover, to avoid nanomaterial pollution, scientists have investigated new materials and chemical compounds which are biodegradable and eco-compatible, such as some biopolymers (i.e., lignin, ${ }^{13,14}$ chitosan, ${ }^{15}$ and alginate $\left.{ }^{16}\right)$. These biopolymers have been already used for nanosystem applications in several sectors of agriculture. In particular, lignin seems to be particularly promising for nanometer containers, since it can be produced as by-product by several industrial activities and is not toxic. ${ }^{17,18}$ In addition, the opportunity to tag biopolymer nanovectors with optically traceable markers could allow monitoring nanomaterials and associated chemicals in plant tissues, ${ }^{19,20}$ as well as their tracking in the ecosystem. This could represent a relevant further advancement and, at the same time, a powerful tool to explain the mechanism of nanovector entrance in plants, as well as its distribution in their different organs, which is still unclear. Developing new nanosystems marked with an appropriate dye would make it possible to track the pathway of nanovectors in plants from the roots to the leaves, through the stem. ${ }^{21}$ In this paper, a new methodology of lignin nanocapsules (NCs) traceability is described in E. tef (Zuccagni) Trotter seedlings (Teff) (Poaceae). The choice of $E$. tef use in the experiments depends on two reasons. The first one is that $E$. tef represents a model plant easy to cultivate and extremely resistant to different conditions. The second one is that this species has an important commercial interest since it represents one of the most cultivated cereals in Ethiopia considering that its production represents the $20 \%\left(2 \cdot 10^{6} \mathrm{t}\right)$ of the total. ${ }^{22,23}$ The tracking method, here described, shows for the first time the use of the fluorochrome Fluorol Yellow 088 (FY088), which was loaded in NCs (fNCs), for tracking lignin NCs in plants. This fluorochrome enabled NC tracking in plantlets through optical microscopy. Moreover, two administration methodologies were tested in order to understand the most efficient way of fNCs entrance in plant tissues.

\section{Materials and Methods}

\section{Materials}

Kraft Lignin (alkali lignin, low sulphur, CAS number 8068-051), acetone and $\mathrm{NaOH}$ were purchased by Sigma Aldrich and used as received. Common olive oil was used for NC preparation. (FY088: 2,8-dimethylnaphtho[3,2,1-k1]xanthene) was provided by Sigma. Milli-Q filtered water was obtained from a Millipore system $\left(\mathrm{M} \Omega \cdot \mathrm{cm}\right.$ at $\left.25^{\circ} \mathrm{C}\right)$. E. tef seeds were purchased in the Tigray Region of Ethiopia.

\section{Nanocapsule preparation}

$\mathrm{NaOH}$ was dissolved in MilliQ water to obtain a solution with a $\mathrm{pH}$ of 12.5. The obtained alkali solution was used to dissolve the lignin powder at a concentration of $1 \% \mathrm{w} / \mathrm{v}$. The final $\mathrm{pH}$ after lignin dissolution was 10.5 . FY088 was diluted in olive oil $(0.1 \%$ $\mathrm{w} / \mathrm{v})$ and then added to acetone, 1:1 (v/v) ratio. Once obtained an olive oil/acetone emulsion, $300 \mu \mathrm{L}$ of this volume was added to $3 \mathrm{~mL}$ of lignin alkali water. Then, high-intensity ultrasound technology was used to emulsify the oil phase in the aqueous medium and to facilitate the fluorochrome encapsulation inside the nanocapsules (Figure 1). For this purpose, the oil/water emulsions were sonicated by Branson 450 Digital Sonifier at the $50 \%$ of the total power $(400 \mathrm{~W})$, for $100 \mathrm{~s}(1 \mathrm{~s}$ pulse on and $1 \mathrm{~s}$ pulse off, to avoid overheating). After ultrasound application, the lignin nanocapsules labeled with FY088 (fNCs) were put in contact with E. tef seedlings.

\section{Nanocapsule treatment}

E. tef seeds were laid down in germination dishes on blotting paper soaked by water. On the $14^{\text {th }}$ day, the seedlings were transferred in Petri dishes to be treated with $\mathrm{fNCs}$ for $24 \mathrm{~h}$. Two different treatments were performed as it is shown in Figure 2: Teff plantlets were soaked with pure nanocapsules solution as it was prepared or laid down on the blotting paper soaked with $\mathrm{fNC}$ solution diluted 1:1 with water.

\section{Microscope analysis}

After $24 \mathrm{~h}$, plantlets were sectioned with Cryo-Cut Microtome $\left(-20^{\circ} \mathrm{C}\right)$ to obtain longitudinal and transverse $20-30 \mu \mathrm{m}$ thick sections. The sections were mounted on microscope slides. A Wild Heerbrugg stereomicroscope and a Leitz DMRB light and fluorescence microscopy with an excitation wavelength of 450-480 nm, equipped with a Nikon DS-5M digital camera were used for microscopic examinations.

\section{Results}

It is generally acknowledged, in laboratory practices that FY088 is an excellent fluorescent marker for lipids staining in fresh tissues. ${ }^{24-26}$ This specific property is the reason why FY088 was chosen in the present study. Indeed, by dissolving it in olive oil, the fluorochrome efficiently dyed the lipid phase. This phase was added to the hydrophilic component in which lignin powder had been previously dissolved at $\mathrm{pH}=12.5$. Ultrasound sonication promoted the formation of an oil/water emulsion, thus improving the entrance of the FY088 present in the lipid phase and stabilizing the fluorochrome inside the lignin shell by hydrophobic repulsion. The resulting lignin NCs loaded with FY088 are shown in Figure 3. These lignin NCs were characterized and found to be a fairly homogenous distribution in size. ${ }^{17}$ The average diameter of loaded lignin NCs was about 200-250 nm while it was in the range of $300 \mathrm{~nm}$ 
for the empty nanoparticles. Fluorochorme nanocapsules were tested on E. tef seedlings to track their entrance in plant tissues. E. tef seedlings were grown in germination chambers for 14 days until they presented a well-developed leaf (Figure 4A) and root (Figure 4B). The seedlings were treated with two different methodologies: the first sample plantlets were soaked with a pure $\mathrm{fNC}$ solution and the second group of plantlets was ordered on blotting paper and wet with a diluted $\mathrm{fNC}$ solution in water. After $24 \mathrm{~h}$, the entrance of fNCs was detected through optical microscope observations and compared with the controls (Figure 4 C,D). After $24 \mathrm{~h}$, fNCs entered inside Teff plantlets through the roots, reaching the xylem vessels (Figure $4 \mathrm{~F}, \mathrm{H}$ ) and they were able to move inside the seedlings, without any forcing from the outside (i.e., magnets). ${ }^{27}$ Moreover, they rapidly arrived to leaf tissues driven by water flow, as it is shown in Figure 4 G,E. Using FY088, it was possible to track the NC path inside Teff tissues, from the roots, through the stem to the leaf (Figure $4 \mathrm{G}, \mathrm{E}$ ). When the fNCs reached the xylem vessels, they were observed inside the vessels and, in large quantity, lining along the vessels walls (Figure 4F). Comparing the two methodologies of administration, the most effective one was found the procedure featuring the immersion of Teff roots in $\mathrm{fNC}$ solution, since the number of $\mathrm{fNCs}$ into the xylem vessels of the stem was higher than that found in the second groups of plantlets disposed on blotting paper soaked with fNCs diluted in water $1: 1$.

\section{Discussion}

One of the main questions about drug administration to plants via $\mathrm{NCs}$ is the route of entrance of the NCs themselves. In this regard, relevant topics are both the penetration route through the plant tegumental tissues towards the internal tissues and the permeation through the wall and eventually plasma membranes towards the cell cytoplasm. To solve this problem, we have designed a new method for tracking lignin NCs in vivo with a fluorescent dye.

Both methods (roots directly in contact with NCs and roots in contact with blotting paper soaked with NCs) were found to be effective, even if the direct exposure to NCs appeared to yield quantitatively better results: more NCs were observed inside. The number of penetrated NCs could be easily detected thanks to the use of the fluorochrome. Concerning the penetration of NCs, several aspects must be considered: the penetration through the external teguments may occur either directly through the walls in the apical roots/root hairs or through stomata in the aerial part of the plant. The direct crossing of the cuticle may be possible for hydrophobic NCs. ${ }^{17}$

Penetration in internal tissues may occur through the apoplastic route (pecto-cellulosic cell walls) and/or the symplastic route through plasmodesmata, while the final entering of the cells implies a crossing of both the pecto-cellulosic wall and the plasma membrane. The Size Exclusion Limit (SEL) of plasmodesmata does not appear compatible with the penetration of NCs of the dimension employed here (200-500 nm) since typical SEL are about $1 \mathrm{~nm}^{28,29}$ even if SEL is a dynamic property ${ }^{30}$ and viruses are able to cause an increase of the SEL itself $^{31}$ while callose may reduce it. The crossing of the pecto-cellulosic cell walls can be done by larger particles. For instance Poly(lactic-co-glycolic) up to $50 \mathrm{~nm}$ nanoparticles can cross the walls, while even nanoparticles up to $500-600 \mathrm{~nm}$ can cross the plasma membranes. ${ }^{32}$

The penetration of NCs inside the xylem vessels and their translocation upwards also implies that NCs are able to cross a lignin wall and that they can be transported by the water flux inside the xylem. We observed the abundant presence of the NCs at the level of the lignified walls of the xylem vessels and even their translocation inside the xylem. In the leaves, the NCs appeared widely spread in the parenchyma.

Previously, other stainings have been used for tracking nanoparticles, such as Alcian Blue in hyaluronic-acid-based nanoparticles in muscle cells to be visualized at transmission electron microscopy, ${ }^{33}$ while our method appears to be the first used in plants for tracking lignin NCs with a fluorescent microscope in plants.

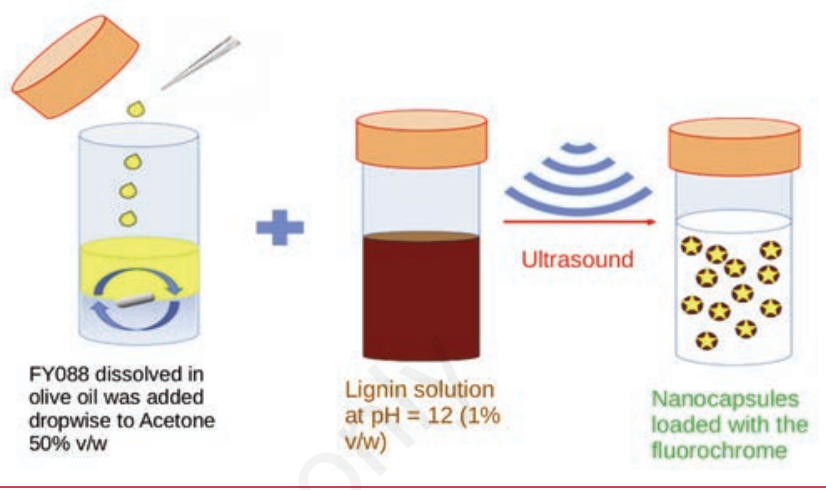

Figure 1. Scheme of fluorescent nanocapsule preparation.

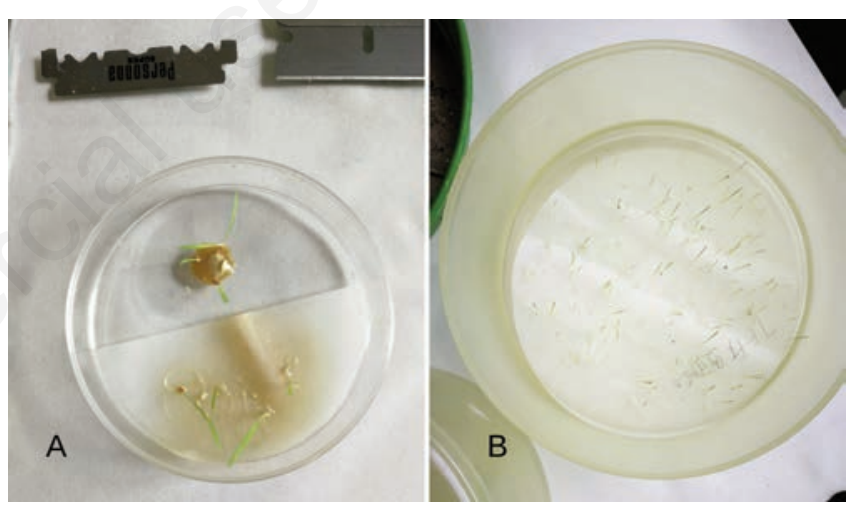

Figure 2. A) E. tef seedlings in germination dish. B) The two methods of $\mathrm{fNC}$ administration to $E$. tef seedlings.

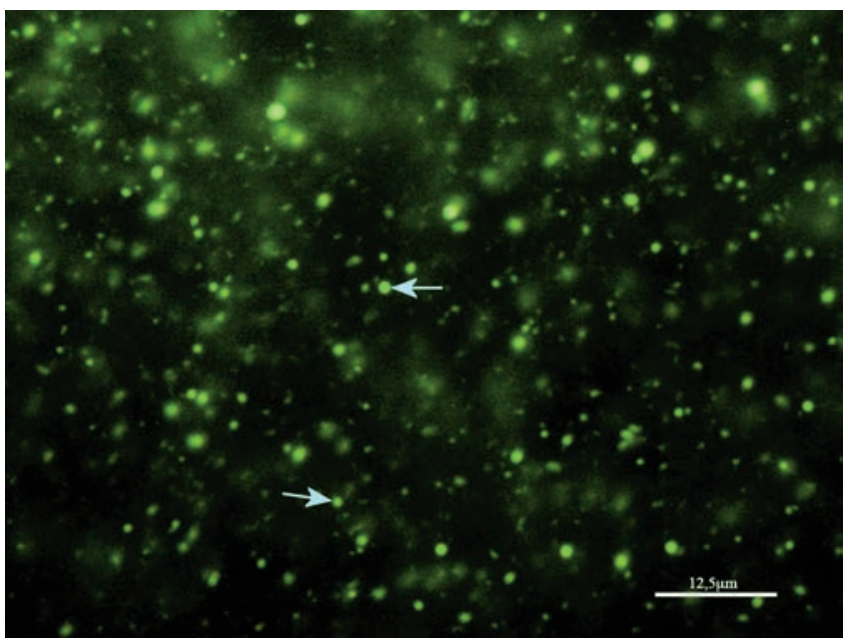

Figure 3. Fluorescence image of lignin nanocapsules loaded with FY088. 
The use of the fluorochrome-charged lignin NCs made it possible to assess the penetration of NCs inside the plant and to identify the best way of administration. It was also possible to show that the NCs reached the xylem vessels in the root and were translocated upwards towards the leaves. In the xylem, a high number of NCs lined the xylem cells at the wall level, hence we can hypothesize that the NCs preferentially follow the walls during their penetration and are also apparently able to follow the lignified walls present in the xylem vessels. The use of the fluorochrome for tracking the NCs penetration route may be extended also to NCs composed of matrixes different from lignin, provided that they are able to store hydrophobic dyes as Fluorol Yellow 088. Further development will include the investigation of lignin NCs in plants with transmission electron microscope, in order to understand their interactions with the cell walls and organules.
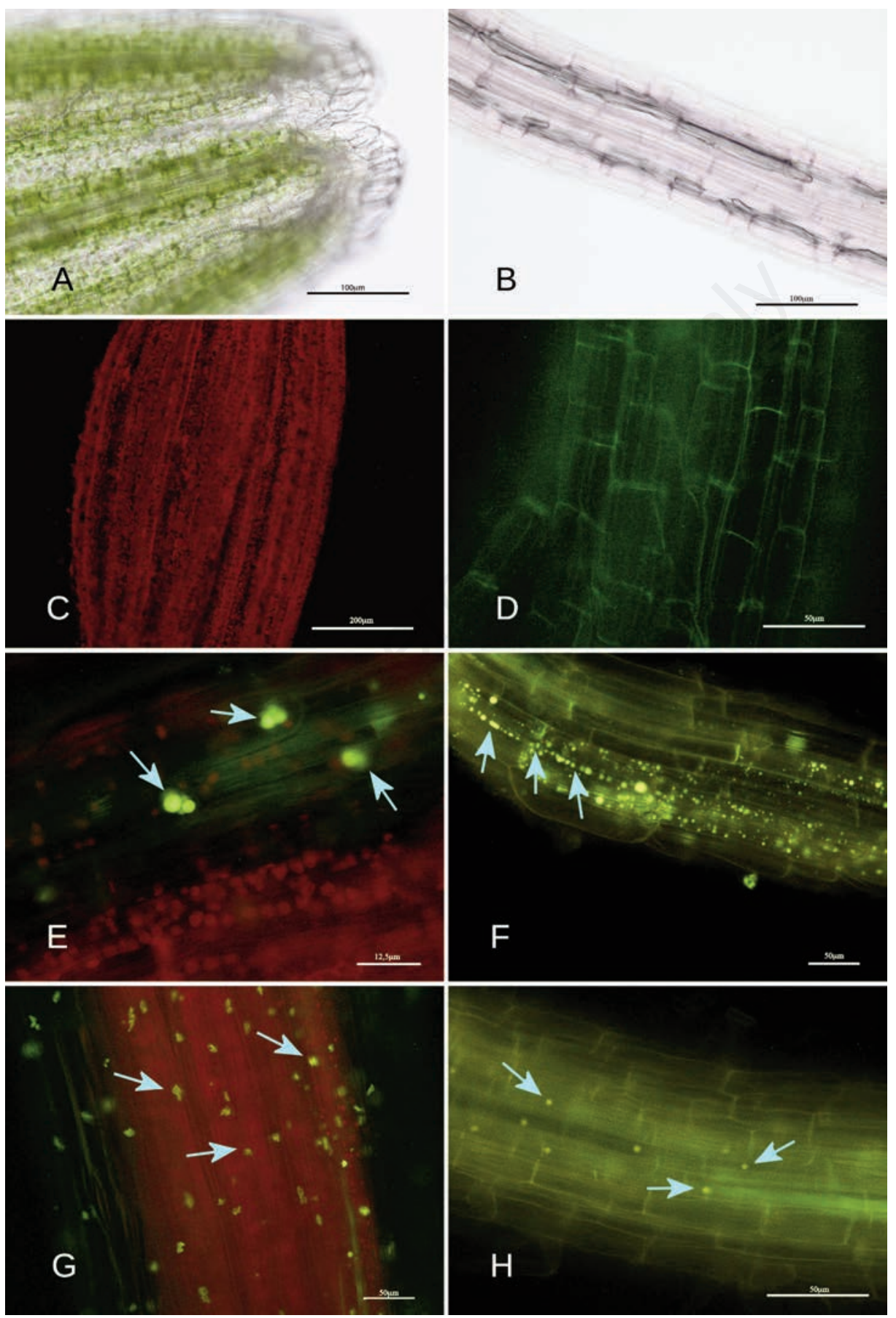

Figure 4. A) Images of a longitudinal sections of Teff leaf. B) Longitudinal section of root. C) Teff leaf without treatment. D) Longitudinal sections of Teff root without treatment, only autofluorescence; E) Teff leaf after $24 \mathrm{~h}$ treatment in pure NCs (some indicated with arrows) loaded with FY088. F) Teff root after $24 \mathrm{~h}$ treatment in pure NCs loaded with FY088; many NCs are lining the xylem walls (arrows). G) Teff leaf after $24 \mathrm{~h}$ treatment on blotting paper soaked with NCs (some indicated with arrows) loaded with FY088. H) Teff root after 24 $h$ treatment in blotting paper soaked with NCs (some indicated with arrows) loaded with FY088. 


\section{Acknowledgments}

This investigation was supported by the CSET, Centro Studi Erbario Tropicale of the University of Florence, Italy.

\section{References}

1. Vurro M, Miguel-Rojas C, Pérez-de-Luque A. Safe nanotechnologies for increasing the effectiveness of environmentally friendly natural agrochemicals. Pest Manag Sci 2019;75:2403-12. doi: 10.1002/ps.5348.

2. Maruyama CR, Guilger M, Pascoli M, Bileshy-José N, Abhilash PC, Fraceto LF, et al. Nanoparticles based on chitosan as carriers for the combined herbicides imazapic and imazapyr. Sci Rep 2016;6:19768. doi: 10.1038/srep19768.

3. Kumar S, Bhanjana G, Sharma A, Sidhu MC, Dilbaghi N. Synthesis, characterization and on field evaluation of pesticide loaded sodium alginate nanoparticles. Carbohydr Polym 2014;101:1061-7.

4. Clemente I, Menicucci F, Colzi I, Sbraci L, Benelli C, Giordano C, et al. Unconventional and sustainable nanovectors for phytohormone delivery: Insights on Olea europaea. ACS Sustainable Chem Eng 2018;6:15022-31. doi: 10.1021/acssuschemeng.8b03489.

5. Pascoli M, Jacques M T, Agarrayua DA, Avila DS, Lima R, Fraceto LF. Neem oil based nanopesticide as an environmentally-friendly formulation for applications in sustainable agricul-ture: An ecotoxicological perspective. Sci Total Environ 2019;677:57-67. doi: 10.1016/j.scitotenv.2019. 04.345 .

6. Ruffini CM, Cremonini R. Nanoparticles and higher plants. Caryologia 2009;62:161-5. doi: 10.1080/00087114.2004. 10589681.

7. Wang P, Lombi E, Zhao F, Kopittke PM. Nanotechnology: a new opportunity in plant sciences. Trends Plant Sci 2016;21:699-712. doi: 10.1016/j.tplants.2016.04.005.

8. Handy RD, von der Kammer F, Lead JR, Hassellöv M, Owen $\mathrm{R}$, Crane M. The ecotoxicology and chemistry of manufactured nanoparticles. Ecotoxicology 2008;17:287-314. doi: 10.1007/s10646-008-0199-8.

9. Owen R, Handy R. Formulating the problems for environmental risk assessment of nanomaterials. Environ Sci Technol 2007;41:5582-8. doi: 10.1021/es072598h.

10. Soh L, Eckelman MJ. Green solvents in biomass processing. ACS Sustainable Chem Eng 2016;4:5821-37.

11. Klöpffer W, Curran MA, Frankl P, Heijungs R, Köhler A, Olsen SI. Nanotechnology and life cycle assessment. Synthesis of results obtained at a workshop, Washington DC 2-3 October 2006. European Commission, DG Research, jointly with the Woodrow Wilson International Center for Scholars.

12. Ettrup K, Kounina A, Hansen SF, Meesters JAJ, Vea EB, Laurent A. Development of comparative toxicity potentials of $\mathrm{TiO} 2$ nanoparticles for use in life cycle assessment. Environ Sci Technol 2017;51:4027-37.

13. Tortora M, Cavalieri F, Mosesso P, Ciaffardini F, Melone F, Crestini C. Ultrasound driven assembly of lignin into microcapsules for storage and delivery of hydrophobic molecules, Biomacromolecules 2014;15:1634-43. doi: 10.1021/bm5000 15j.

14. Yiamsawas D, Baier G, Thines E, Landfestera K, Wurm FR. Biodegradable lignin nanocontainers. RSC Adv 2014;4: 11661-3.

15. Pereira AES, Sandoval-Herrera IE, Zavala-Betancourt SA,
Oliveira HC, Ledezma-Pérez AS, Romero $J$ et al. $\gamma$ Polyglutamic acid/chitosan nanoparticles for the plant growth regulator gibberellic acid: Characterization and evaluation of biological activity. Carbohydr Polym 2017;157: 1862-73.

16. Kumar S, Bhanjana G, Sharma A, Sidhu MC, Dilbaghi N. Synthesis, characterization and on field evaluation of pesticide loaded sodium alginate nanoparticles. Carbohydr Polym 2014;101:1061-7.

17. Iravani S and Varma RS. Greener synthesis of lignin nanoparticles and their applications. Green Chem 2020;22:61236. doi: 10.1039/C9GC02835H.

18. Mishra PK, Wimmera R. Aerosol assisted self-assembly as a route to synthesize solid and hollow spherical lignin colloids and its utilization in layer by layer deposition. Ultrasonics Sonochemistry 2017;35:45-50. doi: 10.1016/j.ultsonch.2016.09.001.

19. Wild E, Jones KC. Novel method for the direct visualization of in vivo nanomaterials and chemical interactions in plants. Environ Sci Technol 2009;43:5290-4.

20. Falsini S, Clemente I, Papini A, Tani C, Schiff S, Salvatici $\mathrm{MC}$, et al. When sustainable nano-chemistry meets agriculture: lignin nanocapsules for bioactive compounds delivery to plantlets. ACS Sustainable Chem Eng 2019;7:19935-42. doi: 10.1021/acssuschemeng.9b05462.

21. Dong R, Li Y, Li W, Zhang H, Liu Y, Ma L, et al. Recent developments in luminescent nanoparticles for plant imaging and photosynthesis. J Rare Earths 2019;37:903-15. doi: 10.1016/ j.jre.2019.04.001.

22. Ketema S. Tef Eragrostis tef (Zucc.) trotter promoting the conservation and use of underutilized and neglected crops. 12. Institute of Plant Genetics and Crop Plant Research, Gatersleben/International Plant Genetic Resources Institute, Rome; 1997;p.7-10.

23. Central Statistical Authority. Agricultural sample survey, 1996/97. Report on the area and production for majorcrops. Private peasant holdings. Statistical Bulletin No. 171. Central Statistical Authority, Addis Ababa; 1997.

24. Brundrett MC, Kendrick B, Peterson CA. efficient lipid staining in plant material with Sudan Red 7B or Fluoral Yellow 088 in polyethylene glycol-glycerol, biotechnic \& histochemistry 1991;66:111-6. doi: 10.3109/10520299109110562.

25. Soukup A. Selected simple methods of plant cell wall histochemistry and staining for light microscopy. In: Žárský V, Cvrčková F, editors. Plant cell morphogenesis. Vol 1080. Humana Press, Totowa; 2014. p. 25-40.

26. Mosti S, Ross Friedman C, Piccolin F, Di Falco P, Papini A. The unusual tegumental tissues of the Lunaria annua (Brassicaceae) seed: a developmental study using light and electron microscopy. Flora 2012;207:828-37. doi: 10.1016/j.flora.2012.09.008.

27. González-Melendi P, Fernández-Pacheco R, Coronado MJ, Corredor E, Testillano PS, Risueño MC, et al. Nanoparticles as smart treatment-delivery systems in plants: Assessment of different techniques of microscopy for their visualization in plant tissues. Ann Bot 2008;101:187-95. doi: 10.1093/ $\mathrm{aob} / \mathrm{mcm} 283$.

28. Iglesias VA, Meins F. Movement of plant viruses is delayed in a $\beta$-1,3-glucanase-deficient mutant showing a reduced plasmodesmatal size exclusion limit and enhanced callose deposition. Plant J 2000;21:157-66.

29. Kim JY. Symplasmic intercellular communication through plasmodesmata. Plants 2018;7:23. doi: 10.3390/plants7010 023.

30. Faulkner C. Plasmodesmata and the symplast. Curr Biol 2018;28:R1374-8. 
31. Wolf S, Williams JL, Deom CM, Beachy RN. Movement protein of tobacco mosaic virus modifies plasmodesmatal size exclusion limit. Science 1989;246:377-9.

32. Palocci C, Valletta A, Chronopoulou L, Donati L, Bramosanti M, Brasili E, et al. Endocytic pathways involved in PLGA nanoparticle uptake by grapevine cells and role of cell wall and membrane in size selection. Plant Cell Rep 2017;36:1917-28.

33. Carton F, Repellin M, Lollo G, Malatesta M. Alcian blue staining to track the intracellular fate of hyaluronic-acidbased nanoparticles at transmission electron microscopy. Eur J Histochem 2019;63:3086. doi: 10.4081/ejh.2019.3086.

Received for publication: 16 January 2020. Accepted for publication: 16 March 2020.

This work is licensed under a Creative Commons Attribution-NonCommercial 4.0 International License (CC BY-NC 4.0).

(C) Copyright: the Author(s), 2020

Licensee PAGEPress, Italy

European Journal of Histochemistry 2020; 64:3112

doi:10.4081/ejh.2020.3112 